\title{
SWITCHED UNIFORM SCALAR QUANTIZATION ADAPTED TO MEAN AND VARIANCE FOR SPEECH CODING
}

\author{
UDC (621.391:004.383.3)
}

\author{
Goran Petković ${ }^{1}$, Zoran Perić ${ }^{2}$, Vladimir Despotović $^{3}$ \\ ${ }^{1}$ College of Applied Studies, Vranje, Republic of Serbia \\ ${ }^{2}$ University of Niš, Faculty of Electronic Engineering, Department of \\ Telecommunications, Niš, Republic of Serbia \\ ${ }^{3}$ University of Belgrade, Technical Faculty in Bor, Bor, Republic of Serbia
}

\begin{abstract}
Average power and variance are widely used in adaptation techniques in signal coding. A speech signal is usually assumed to be zero-mean; thus an average signal power is equal to the signal variance. However, this assumption is valid only for longer signals with a large number of samples. When the signal is divided into frames (especially if the number of samples within the frame is small) the speech signal within the frame may not be zero-mean. Hence, frame-by-frame adaptation to signal mean might be beneficial. A switched uniform scalar quantizer with adaptation to signal mean and variance is proposed in this paper. The analysis is performed for different frame lengths and the results are compared to an adaptive uniform quantizer that uses adaptation only to average signal power, showing an improved performance. Signal to quantization noise ratio $(S Q N R)$ is used as a performance measure.
\end{abstract}

Key words: adaptive quantization, speech coding, signal processing

\section{INTRODUCTION}

A speech signal is considered to be nonstationary since its parameters are changing with respect to time. However, its frequency content varies slowly with time, hence it can be assumed that the speech signal analysed over short-duration intervals (frames) is nearly stationary (quasi-stationary) [1]. Many techniques in signal processing exploit these local statistic characteristics of the signal, obtained when it is analysed frame-by-frame [2-4].

The average signal power is commonly used statistical parameter for adaptation in adaptive signal quantization [5, 6]. When the signal is zero-mean, which is often assumed in speech [7], the average power is equal to the signal variance. However, this assumption

Received August 26, 2017

Corresponding author: Goran M. Petković

College of Applied Studies, Filipa Filipović 20, 17500 Vranje, Republic of Serbia,

E-mail: goranpetko@yahoo.com 
might not be valid when the signal is divided into frames (especially if the number of samples within the frame is small); hence the average signal power and variance will differ. This signal characteristic can be exploited for designing the adaptive quantizer.

This paper proposes a frame-by frame adaptive uniform scalar quantizer with adaptation to signal mean and variance. The switched quantization techniques are used, where the dynamic range of the signal is divided into a number of subranges, with one quantizer designed for each subrange. A proper quantizer is chosen based on the signal variance for each consecutive frame. In this way high and stable SQNR may be obtained in a wide range of signal variances. The results are compared with the standard uniform scalar quantizer that uses adaptation only to average signal power. SQNR is used as a performance measure.

The remaining of the paper is organized as follows. Section 2 presents statistical parameters used for adaptation in adaptive quantization techniques with the analysis performed for different frame lengths and various speech signals. In Section 3 a brief overview of the uniform scalar quantization is given. In Section 4 the adaptive switched uniform quantizer with adaptation to signal mean and variance is designed. In Section 5 the obtained experimental results using a real speech signal are presented and discussed. Finally, concluding remarks are given in Section 6.

\section{STATISTICAL PARAMETERS FOR SignAl ADAPTATION IN ADAPTIVE QUANTIZATION}

Adaptive quantization attempts to adapt the quantizer to the varying input statistics in order to achieve a better performance [8-10]. Average signal power and variance are the most frequently used statistical parameters for adaptation, where the average signal power can be determined as:

$$
P=\frac{1}{N} \sum_{i=1}^{N} x_{i}^{2},
$$

and variance as:

$$
V=\frac{1}{N} \sum_{i=1}^{N}\left(x_{i}-\mu\right)^{2},
$$

where $x_{i}, i=1, \ldots, N$ defines the discretized speech signal with $N$ samples and $\mu$ is the signal mean:

$$
\mu=\frac{1}{N} \sum_{i=1}^{N} x_{i} .
$$

Alternatively, one can use Route Mean Square (RMS) of the signal defined as the square root of the signal power:

$$
R M S=\sqrt{P}=\sqrt{\frac{1}{N} \sum_{i=1}^{N} x_{i}^{2}},
$$

and standard deviation, calculated as the squared root of the variance: 


$$
\sigma=\sqrt{V}=\sqrt{\frac{1}{N} \sum_{i=1}^{N}\left(x_{i}-\mu\right)^{2}} .
$$

A speech signal with the duration of 4 seconds is represented in Fig. 1. The signal mean is approximately equal to zero. Comparing (1) and (2) one can note that in that case average signal power and variance are equal. However, if the signal is divided into short-term intervals called frames, than the signal mean within a frame is not equal to zero anymore, which is obvious in Fig. 2, where one frame with the duration of $10 \mathrm{~ms}$ is depicted. Hence, the average signal power determined on a frame deviates from the variance.

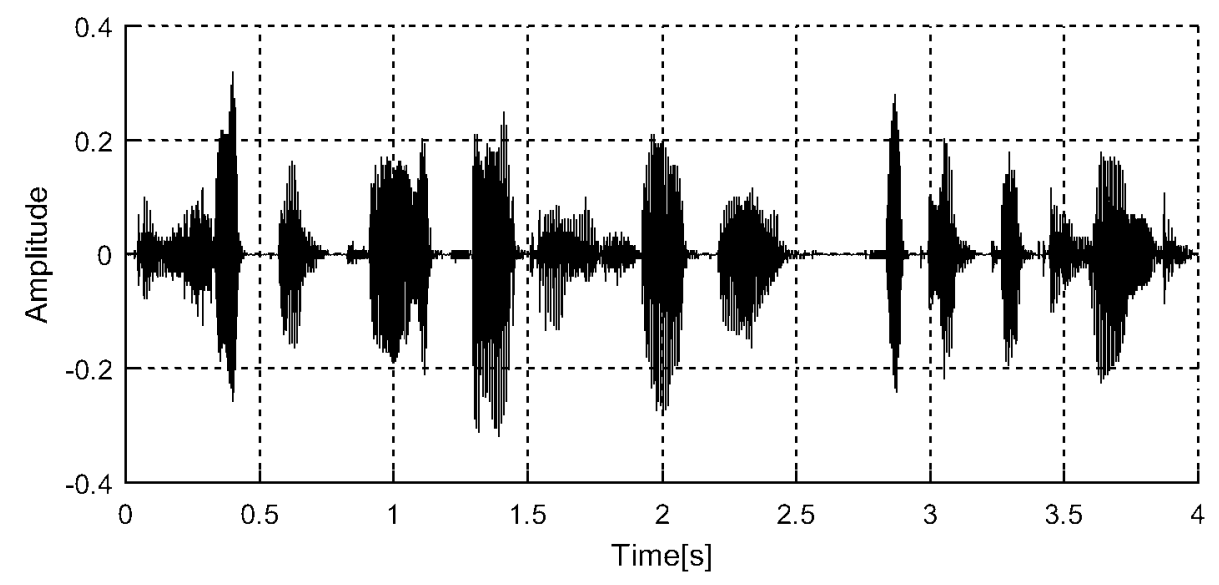

Fig. 1 Speech signal represented in time domain (duration $4 \mathrm{~s}$ )

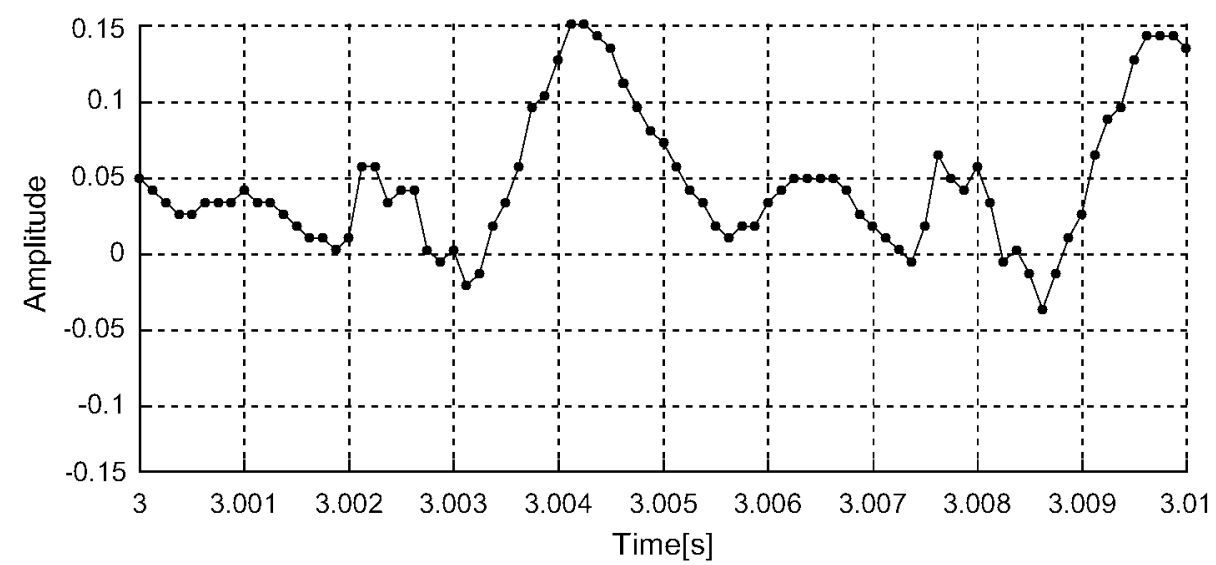

Fig. 2 One frame of the speech signal depicted in Fig. 1 with the duration of $10 \mathrm{~ms}$ 


\subsection{Analysis of average signal power and variance using speech signal}

The analysis is performed using the speech corpus of the International Telecommunication Union - Telecommunication Standardization Sector ITU-T [11]. The language of the corpus is English with 16 speakers in total, 8 of them being males (SP01,..,SP08), and the remaining 8 being females $(\mathrm{SP} 09, \ldots, \mathrm{SP} 16)$. The speech signal is sampled at $8 \mathrm{kHz}$ sampling rate and encoded using 16 bits per sample.

The speech is divided into frames of frame lengths between 10 and 320 samples. Table 1 presents signal power and variance averaged over all speech frames $\left(\overline{P_{F}}\right)$ for different speech signals and different frame lengths. While $\overline{P_{F}}$ is independent of the frame length, $\overline{\sigma_{F}^{2}}$ changes with the frame duration, but remains approximately equal to $\overline{P_{F}}$ for frame lengths between 80 and 320 samples.

Furthermore, the root mean square deviation (Rmsd) of the average signal power from the variance over $s$ frames is determined and presented in Table 1:

$$
R m s d=\sqrt{\frac{1}{s} \sum_{i=1}^{s}\left(P_{F i}-\sigma_{F i}^{2}\right)^{2}} .
$$

The smallest Rmsd is found for 320 sample frame lengths, while the highest value is obtained for the shortest frames (10 samples), which is expected as for longer signals the variance approaches the average signal power.

Table 1 Signal power, variance and Rmsd averaged over all speech frames

\begin{tabular}{|c|c|c|c|c|c|c|c|c|c|c|c|c|c|}
\hline \multirow{3}{*}{$\begin{array}{r}\text { Speech } \\
\text { signal }\end{array}$} & \multirow{3}{*}{$\begin{array}{c}\overline{P_{F}} \\
{\left[\mathrm{x} 10^{-4}\right]}\end{array}$} & & & $\overline{\sigma_{F}^{2}}$ & {$\left[\mathrm{x} 10^{-4}\right]$} & & & \multicolumn{6}{|c|}{ Rmsd $\left[\times 10^{-4}\right]$} \\
\hline & & \multicolumn{6}{|c|}{ Frame length [number of samples] } & \multicolumn{6}{|c|}{ Frame length [number of samples] } \\
\hline & & 10 & 20 & 40 & 80 & 160 & 320 & 10 & 20 & 40 & 80 & 160 & 320 \\
\hline SP 01 & 14.95 & 12.29 & 14.26 & 14.97 & 15.08 & 15.03 & 15.02 & 9.96 & 3.26 & 0.86 & 0.37 & 0.18 & 0.10 \\
\hline SP 02 & & 5.76 & 7.94 & 9.17 & 9.89 & 9.87 & 9.87 & 10.87 & 4.65 & 1.66 & & & \\
\hline $\mathrm{SPC}$ & 1( & 13.13 & 15.28 & 16.33 & 16 & 16 & 16 & 1 & 4.90 & 1.62 & & & 0 \\
\hline SP & 0 & 21.98 & 2 & 27.66 & 2 & 6 & 27 & 22.29 & 6.90 & & & & \\
\hline SP & 22 & 15 & 2 & 28.73 & 2 & 5 & 4 & 40.42 & 12.33 & & & & U \\
\hline & 8 & 69 & 2 & 29.03 & & 29.31 & 29 & 34.89 & 9.76 & & & & \\
\hline SP 07 & 9.79 & 22.40 & 26.52 & 29.50 & 29.90 & 29.93 & 29.89 & 26.67 & 10.60 & 2.41 & 81 & 0.43 & 0.22 \\
\hline SP 08 & 29.20 & 19.64 & 25.52 & 28.92 & 29.31 & 29.34 & 29.33 & 37.02 & 11.91 & 2.47 & 7 & 0 & 0.19 \\
\hline SP 09 & 5.66 & 4.71 & 5.48 & 5.74 & 5.70 & 5.69 & 5.67 & 3.89 & 1.15 & 0.36 & 0.17 & 0.10 & 0.05 \\
\hline SP 10 & 15.71 & 12.60 & 15.01 & 15.97 & 15.86 & 15.80 & 15.79 & 11.36 & 3.57 & 0. & 0 & 0.23 & 0.12 \\
\hline SP 11 & & 1.43 & 2.32 & 2.99 & 2.96 & 2.95 & 2.95 & 3.85 & 1.54 & 0. & 0 & 0.03 & 0.01 \\
\hline SP & & 22.16 & 23.94 & 24.44 & 24.25 & 24. & 24 & 10.53 & 3.59 & & & & \\
\hline & & 14.67 & 23.22 & 27.56 & 27. & 27. & 21. & 33.99 & 11 & & & & \\
\hline SP 14 & 24.79 & 13.41 & 21.23 & 25.15 & 24.95 & 24.90 & 24.85 & 32.21 & 10.44 & 1.08 & & 0.25 & 0.13 \\
\hline SP 15 & 23.40 & 12.24 & 19.15 & 23.84 & 23.60 & 23.53 & 23.49 & 32.90 & 11.95 & 1.14 & & 0.25 & 0.13 \\
\hline SP 16 & 23.52 & 10.42 & 18.71 & 23.96 & 23.71 & 23.64 & 23.63 & 36.39 & 12.47 & 1.07 & 0.51 & 0.24 & 0.12 \\
\hline
\end{tabular}

Figures 3 and 4 show the normalized distribution of the average power and variance for frame lengths of 10 samples and 160 samples, respectively. While these distributions are clearly different for small frames (Fig. 3), they show almost a perfect match for longer speech frames (Fig. 4), again confirming the conclusions drawn from Table 1. 


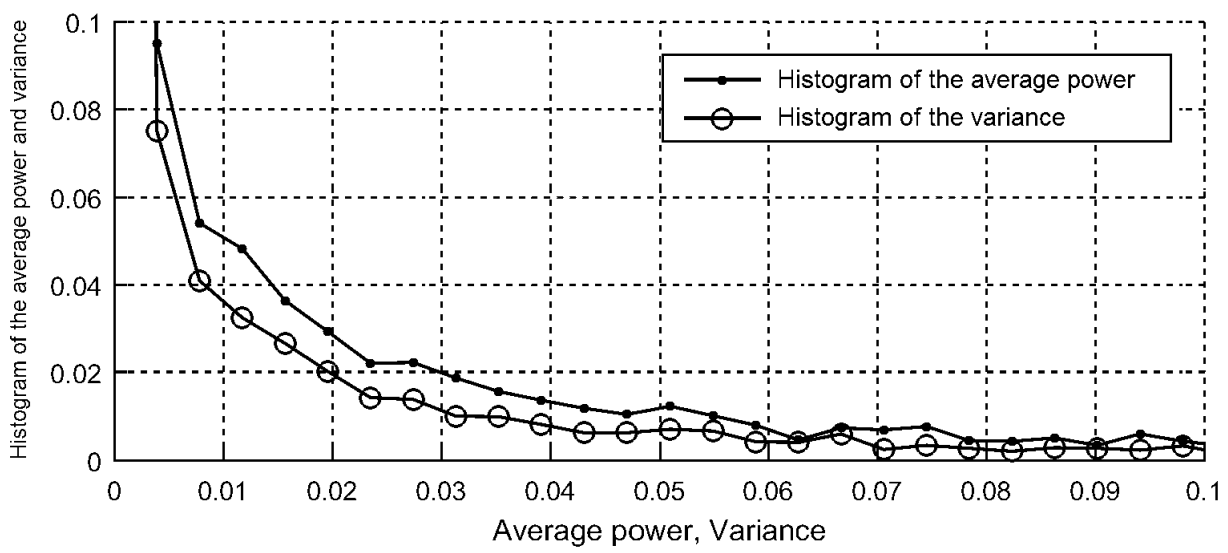

Fig. 3 Normalized distribution of average power and variance for frame lengths of 10 samples (speaker SP01)

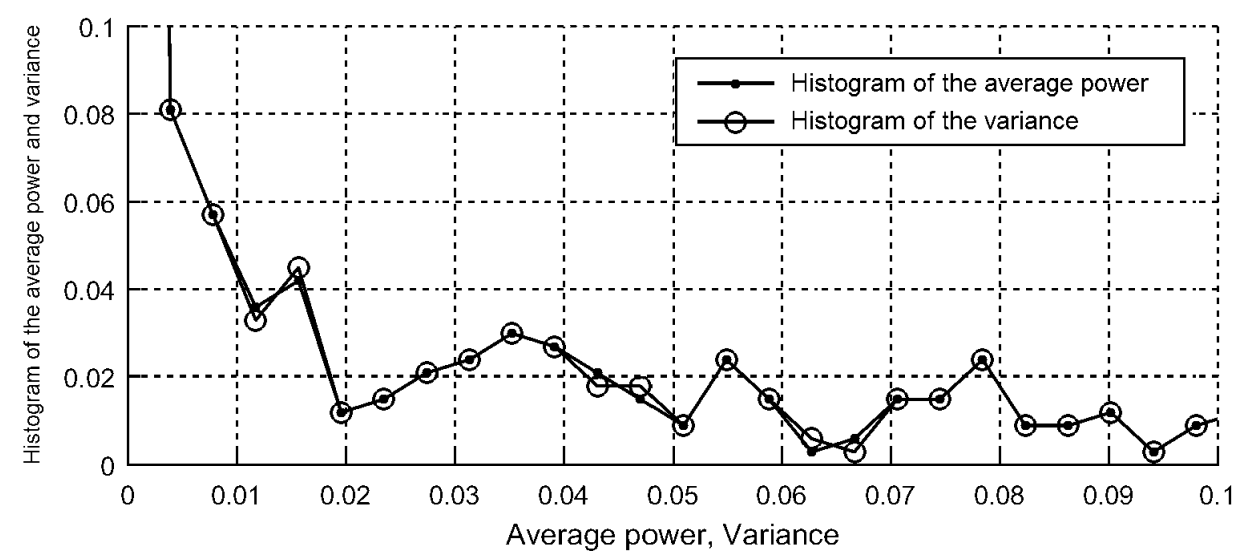

Fig. 4 Normalized distribution of average power and variance for frame lengths of 160 samples (speaker SP01)

\section{UNIFORM SCALAR QUANTIZER}

The uniform scalar quantizer $Q$ is specified by $N$ quantization levels $\left\{y_{1}, y_{2}, \ldots, y_{N}\right\}$ and $N+1$ decision thresholds $\left\{x_{0}, x_{1}, \ldots, x_{N}\right\}$. The quantizer is defined as $Q(x)=y_{i}$, where $x_{i-1}<x \leq x_{i}$ for $i=1,2, \ldots, N$, and $\infty=x_{0}<x_{1}<\ldots<x_{N}=\infty$. The quantized signal takes value $y_{i}$ when the original signal belongs to the cell $S_{i}=\left(x_{i-1}, x_{i}\right]$ for $i=1,2, \ldots, N$.

Distortion is a measure of irreversible error introduced during the quantization process using $N$ quantization levels and can be expressed as a sum of granular distortion $D_{g}$ and overload distortion $D_{o}$ : 


$$
D=D_{g}+D_{o} .
$$

Each quantizer is designed for a predefined support region. The distance between the quantization levels is referred to as its granularity, and the introduced error is granular distortion:

$$
D_{g}=\sum_{i=2}^{N-1} \int_{x_{i-1}}^{x_{i}}\left(x-y_{i}\right) p(x) d x
$$

For a large $N$ the equation (8) can be approximated as:

$$
D_{g}=\frac{\Delta^{2}}{12} \int_{-x_{\max }}^{x_{\max }} p(x) d x,
$$

where $x_{\max }$ represents the bounds of the quantizer's support range and $p(x)$ is probability density function (pdf).

Whenever the input exceeds the supported range $\left(x>x_{\max }\right.$ or $\left.x<-x_{\max }\right)$, the clipping occurs, and the error introduced by this clipping is referred to as overload distortion $[12,13]$ :

$$
D_{o}=2 \sum_{i=N-1}^{\infty} \int_{x_{i-1}}^{x_{i}}\left(x-y_{N}\right) p(x) d x .
$$

The balance between granular distortion and overload distortion has to be found by the proper choice of $x_{\max }$, as reducing the granular distortion may increase the overload distortion, and vice versa.

Speech signal is modeled by Laplacian pdf, as it was shown that for frames longer than $5 \mathrm{~ms}$ speech signal distribution tends to Laplacian distribution [14]:

$$
p(x)=\frac{1}{\sqrt{2} \sigma} e^{\frac{\sqrt{2}|x|}{\sigma}} .
$$

For simplicity, we will assume that the signal is zero-mean, so we can substitute signal power with variance [15]. Granular and overload distortion of the uniform quantizer in that case become:

$$
\begin{gathered}
D_{g}=\frac{c^{2} t^{2} \sigma^{2}}{3 N^{2}}\left(1-e^{-\sqrt{2} c t}\right), \\
D_{o}=e^{-\sqrt{2} c t} \sigma^{2}\left(1+\sqrt{2} \frac{c t}{N}+\left(\frac{c t}{N}\right)^{2}\right),
\end{gathered}
$$

where $t=\sigma_{0} / \sigma, c=x_{\text {max }} / \sigma_{0}$, and $\sigma_{0}^{2}$ is the referent variance [16].

Beside distortion, SQNR is often used as a measure of performance:

$$
\mathrm{SQNR}=10 \log \left(\frac{\sigma^{2}}{D}\right),
$$


given in decibels (dB), where $\sigma^{2}$ is the input signal variance and $D$ is the total distortion, as given in (7).

Fig. 5 shows SQNR in the wide range of signal variances for the uniform scalar quantizer for $N=64, N=128$ and $N=256$ quantization levels. The maximal value of SQNR is determined for $\sigma=\sigma_{0}$.

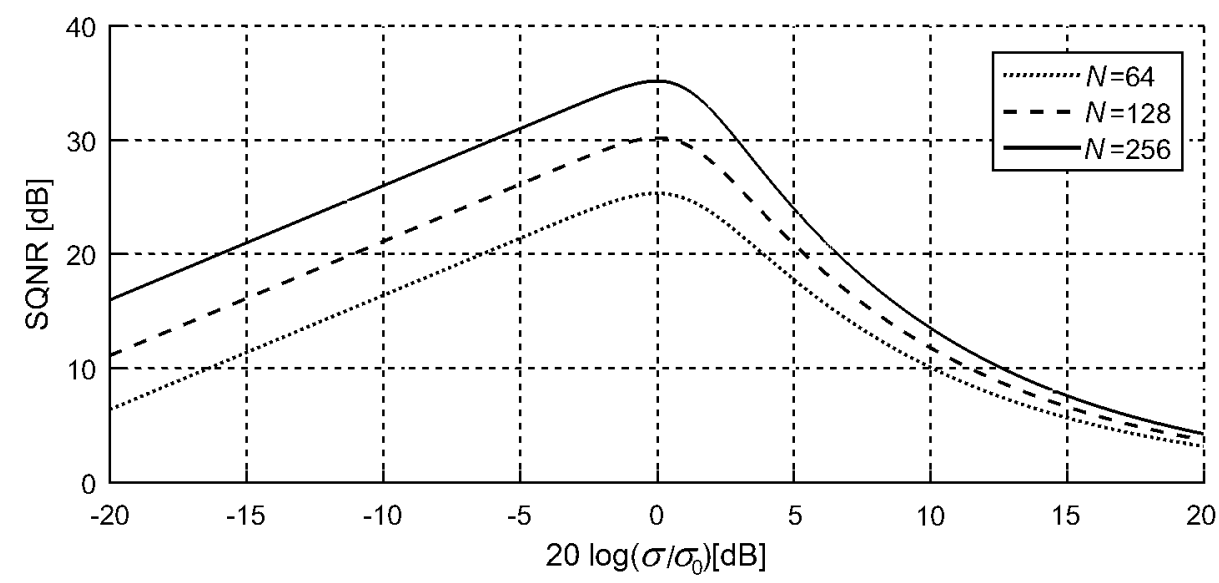

Fig. 5 SQNR of the uniform scalar quantizer with $N=64, N=128$ and $N=256$ quantization levels in the wide dynamic range

The numerical Nelder-Mead method [17-21] was used to determine the optimal parameter $c_{\text {opt }}$ for which SQNR is maximal, and thus the optimal upper bound of the quantizer's support region threshold $x_{\max }^{\mathrm{opt}}$. Table 2 gives an optimal parameter $c_{\mathrm{opt}}$ and a maximal SQNR for various numbers of quantization levels.

Table $2 \mathrm{SQNR}_{\max }$ and $c_{\mathrm{opt}}$ for various numbers of quantization levels $N$

\begin{tabular}{cccc}
\hline$N$ & 64 & 128 & 256 \\
\hline$c_{\mathrm{opt}}$ & 5.30 & 6.15 & 7.02 \\
$\mathrm{SQNR}_{\max }$ & 25.36 & 30.23 & 35.21 \\
\hline
\end{tabular}

\section{SWITCHED UNIForm SCALAR QUANTIZER ADAPTED to SignAL MEAN AND VARIANCE}

The proposed model uses the switched quantization technique, where the dynamic range of the signal is divided into subranges, with one quantizer designed optimally for each subrange. The proper quantizer is chosen based on the signal variance for each consecutive frame, hence the information about the selected subrange has to be sent to the receiver end for each frame. This increases the necessarry bit-rate compared to a single quantizer, and the number of required bits depends on the number of subranges. However, this increase is not significant, as this additional information is sent only once per frame.

Both uniform and logarithmic quantizers can be used in the switched quantization. While the uniform quantizers have lower complexity, the logarithmic quantizers give a 
better performance. Switched quantization ensures high and stable SQNR in a wide range of signal variances.

Fig. 6 presents SQNR for the switched uniform scalar quantization with adaptation to signal variance with $k=1,2$ and 4 quantizers for the same number of subranges. A higher number of subranges obviously leads to smaller changes in SQNR. This quantizer will be used as a baseline for comparison to a model that uses adaptation to both signal mean and variance.

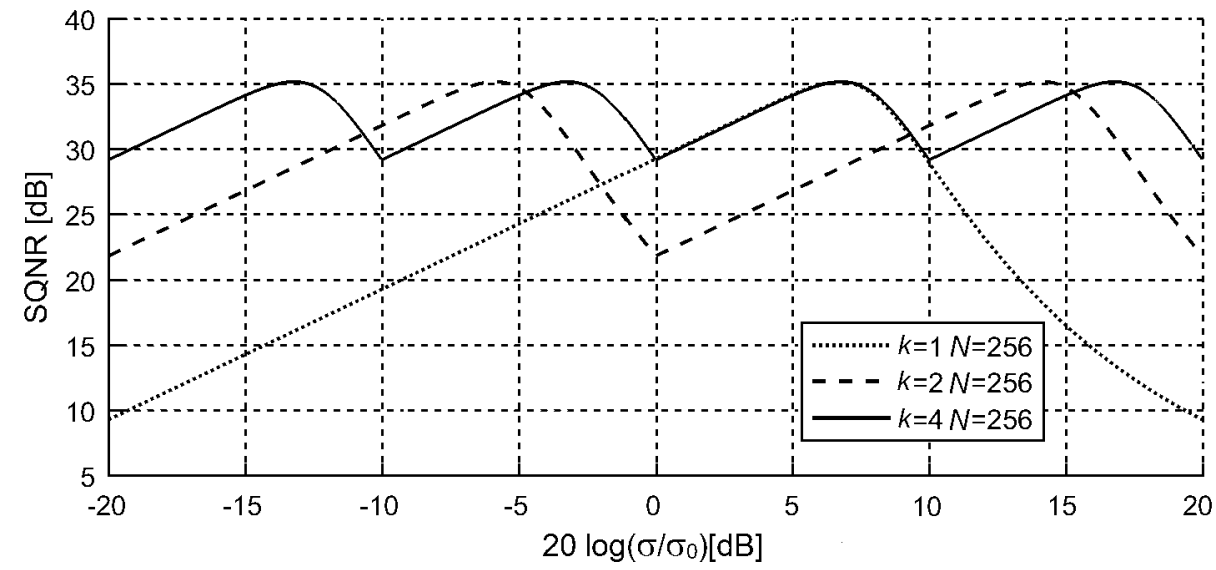

Fig. $6 \mathrm{SQNR}$ for switched uniform quantizer with adaptation to the variance, for $N=256$ quantization levels and different number of quantizers $(k=1, k=2, k=4)$.

The motivation for the design of switched uniform scalar quantizer adapted to signal mean and variance comes from the results of analysis carried out in Section 2, which show that for shorter frames the average signal power deviates from the variance. Instead of using only variance for signal adaptation, which is common in adaptive quantization, we propose using both signal mean and variance.

The block diagram of the proposed quantization technique is given in Fig. 7. The signal is first buffered into frames of length $M$, with samples $x_{(j-1) M+i}, i=1, \ldots, M$, where $j$ denotes the $j$-th frame. For each frame the mean of the samples belonging to that frame is calculated and denoted with $A$. The mean is quantized using the logarithmic $\mu$ law quantizer with $N_{1}$ quantization levels:

$$
c(x)=\frac{\ln \left(1+\mu \frac{|x|}{x_{\max }}\right)}{\ln (1+\mu)} \operatorname{sgn}(x),
$$

Quantized mean $A_{Q}$ is encoded and sent to the receiver end as the side information. In the next step quantized mean $A_{Q}$ is subtracted from each sample within the $j$-th frame:

$$
\hat{x}_{(j-1) M+i}=x_{(j-1) M+i}-A_{Q}, \quad i=1, \ldots, M .
$$


The variance is determined for each $\hat{x}_{(j-1) M+i} i=1, \ldots, M$, and based on this variance one of $k$ available quantizers in the switched quantization scheme is chosen. The quantizers are optimally designed for $k$ variances which are log-uniformly distributed in the dynamic range $B=20 \log \left(\sigma_{\max } / \sigma_{\min }\right)$ :

$$
20 \log \hat{\sigma}_{p}=20 \log \sigma_{\min }+(2 p-1) \frac{B}{2 k}, p=1, \ldots, k,
$$

where $p$ denotes $p$-th subrange. For each subrange an optimal parameter $c_{\mathrm{opt}}$ is determined, to ensure the minimal distortion and maximal SQNR. The parameter $c_{\mathrm{opt}}$ is used to calculate the optimal upper bound of the quantizer's support range.

The selected uniform quantizer with $N$ quantization levels is used for quantization of samples of the current frame $[19,22]$. Beside this, the bit-stream has to include the side information about the selected quantizer and the quantized and encoded sample mean $\left(A_{c}\right)$.

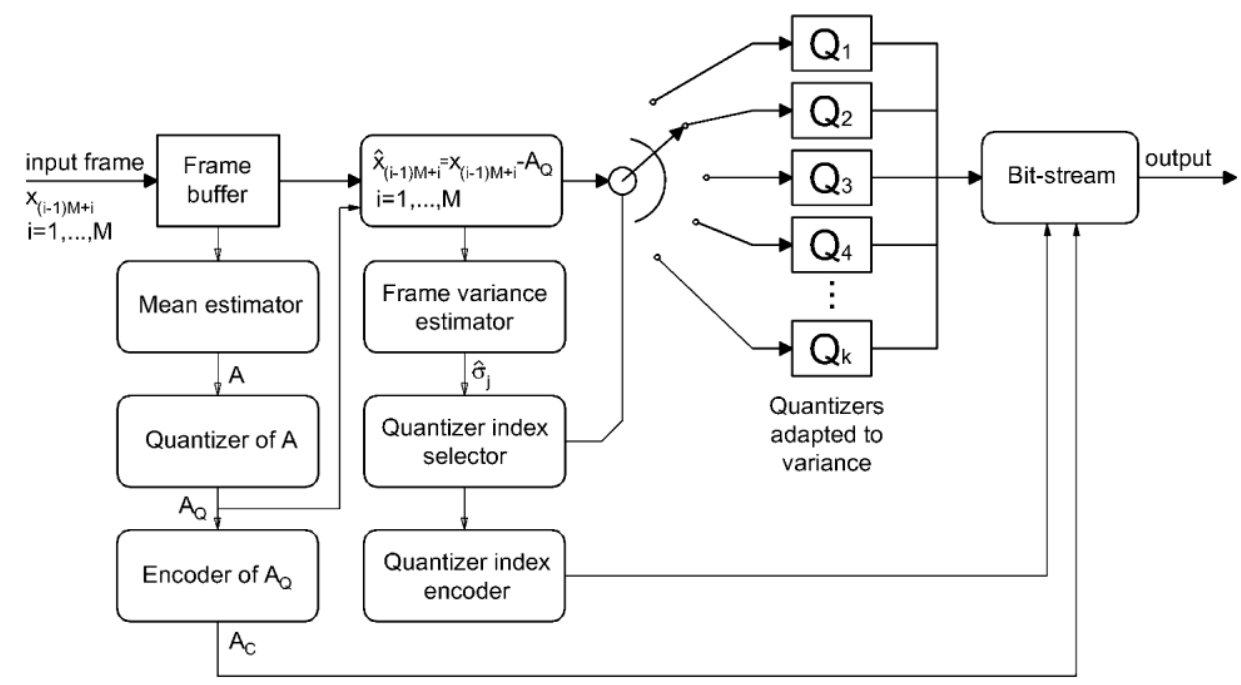

Fig. 7 Block diagram of the switched uniform scalar quantization with adaptation to signal mean and variance

At the reciever end the incoming bit-stream is unpacked, the index of the selected quantizer is decoded and an appropriate inverse quantizer for the selected subrange is selected. Finaly, decoded mean is added to each decoded sample in the frame, as a reverse operation to the one at the transmitter end.

Adaptation to both mean and variance demands the transfer of side information to the receiver end, leading to a total bit-rate:

$$
R=\log _{2} N+\frac{\log _{2} N_{1}}{M}+\frac{\log _{2} k}{M},
$$


where the first term denotes bit-rate for encoding each sample in the frame, the second term carries the information about the quantized mean and the third term carries the information about the selected quantizer (subrange). Obtained bit-rates for different frame lengths are shown in Table 3.

Table 3 Bit-rate $R$ for $N=256$ and $N_{1}=256$ [bits/sample]

\begin{tabular}{cccccccc}
\hline & $\begin{array}{c}\text { Frame length } M \\
\text { [number of samples] }\end{array}$ & 10 & 20 & 40 & 80 & 160 & 320 \\
\hline Number of & 2 & 8.90 & 8.45 & 8.23 & 8.11 & 8.06 & 8.03 \\
quantizers & 4 & 9.00 & 8.50 & 8.25 & 8.13 & 8.06 & 8.03 \\
$k$ & 8 & 9.10 & 8.55 & 8.28 & 8.14 & 8.07 & 8.03 \\
\hline
\end{tabular}

\section{EXPERIMENTAL RESULTS AND DISCUSSION}

The experimental results are obtained using the speech corpus presented in Section 2.1. SQNR was used as a performance measure and the results for the proposed switched uniform quantizer adapted to mean and variance are compared to a standard switched uniform quantizer adapted only to variance.

Four uniform quantizers with $N=256$ quantization levels were used in the switched quantization scheme, which cover the dynamic range $[-20 \mathrm{~dB}, 20 \mathrm{~dB}]$. The sample mean is quantized using the logarithmic quantizer with $N_{1}=256$ quantization levels.

Table 4 presents average SQNR for different frame lengths and various speech signals, both male (SP01,..,SP08), and female (SP09,..,SP16). Switched quantizer adapted to variance is denoted as ' $Q \mathrm{~V}$ ', while the proposed switched quantizer adapted to both mean and variance is denoted as ' $\mathrm{Q}_{\mathrm{M} \& \mathrm{~V}}$ '. One can note that the proposed quantizer outperforms the baseline for all speakers. The improvement is larger for shorter frames, which is expected, as the deviation of the signal power from the variance is more evident in that case. The obtained SQNR is in accordance with the theoretical results presented in Fig. 6.

\section{CONCLUSION}

This paper proposes a switched adaptive uniform scalar quantizer with adaptation to signal mean and variance. The analysis of the average power and the variance have shown that for shorter frames the average power significantly deviates from the variance, as the signal is not zero-mean. This provides motivation for using the signal mean for adaptation, in addition to the variance. In this way, a better performance is obtained, at the cost of slightly increased bit-rate, as the additional information about the signal mean has to be transmitted to the receiver end.

The proposed model is especially convenient for quantization of signals sampled at high sampling frequencies, such as high quality speech, audio and music signals. 
Table 4 Average SQNR for different frame lengths using the switched quantizer adapted to variance $\left(\mathrm{Q}_{\mathrm{V}}\right)$, and the switched quantizer adapted to mean and variance $\left(\mathrm{Q}_{\mathrm{M} \& \mathrm{~V}}\right)$

\begin{tabular}{|c|c|c|c|c|c|c|c|}
\hline \multirow{2}{*}{ Speech signal } & \multirow{2}{*}{$\begin{array}{c}\text { Type of } \\
\text { adaptation }\end{array}$} & \multicolumn{6}{|c|}{ Frame length [number of samples] } \\
\hline & & 10 & 20 & 40 & 80 & 160 & 320 \\
\hline \multirow{2}{*}{ SP 01} & $\mathrm{QV}_{\mathrm{V}}$ & 32.10 & 32.01 & 32.04 & 32.27 & 32.00 & 31.94 \\
\hline & $\mathrm{Q}_{\mathrm{M} \& \mathrm{~V}}$ & 33.66 & 33.08 & 33.08 & 33.44 & 33.18 & 33.10 \\
\hline \multirow{2}{*}{ SP 02} & $\mathrm{QV}_{\mathrm{V}}$ & 32.51 & 32.11 & 32.04 & 31.86 & 31.62 & 31.52 \\
\hline & $\mathrm{Q}_{\mathrm{M} \& \mathrm{~V}}$ & 35.24 & 33.92 & 33.61 & 33.21 & 32.91 & 33.01 \\
\hline \multirow{2}{*}{ SP 03} & $\mathrm{Q}_{\mathrm{V}}$ & 32.47 & 32.18 & 32.43 & 32.39 & 32.20 & 31.70 \\
\hline & $\mathrm{Q}_{\mathrm{M} \& \mathrm{~V}}$ & 34.00 & 33.12 & 33.22 & 33.24 & 33.09 & 32.60 \\
\hline \multirow{2}{*}{ SP03 } & $\mathrm{Q}_{\mathrm{V}}$ & 32.92 & 32.92 & 32.98 & 33.06 & 33.04 & 32.60 \\
\hline & $\mathrm{Q}_{\mathrm{M \& V}}$ & 34.13 & 33.56 & 33.58 & 33.66 & 33.46 & 33.24 \\
\hline \multirow{2}{*}{ SP04 } & $\mathrm{Q}_{\mathrm{V}}$ & 32.66 & 32.40 & 32.30 & 32.41 & 32.46 & 32.35 \\
\hline & $\mathrm{Q}_{\mathrm{M} \& \mathrm{~V}}$ & 35.17 & 33.36 & 32.75 & 32.81 & 32.94 & 32.86 \\
\hline \multirow{2}{*}{ SP05 } & $\mathrm{Q}_{\mathrm{V}}$ & 32.73 & 32.50 & 32.51 & 32.35 & 32.35 & 32.32 \\
\hline & $\mathrm{Q}_{\mathrm{M} \& \mathrm{~V}}$ & 34.78 & 33.33 & 32.89 & 32.76 & 32.84 & 32.85 \\
\hline \multirow{2}{*}{ SP06 } & $\mathrm{Q}_{\mathrm{V}}$ & 33.00 & 32.87 & 32.89 & 32.71 & 32.62 & 32.78 \\
\hline & $\mathrm{Q}_{\mathrm{M} \& \mathrm{~V}}$ & 34.45 & 33.83 & 33.43 & 33.16 & 33.13 & 33.25 \\
\hline \multirow{2}{*}{ SP07 } & $\mathrm{Q}_{\mathrm{V}}$ & 32.83 & 32.82 & 32.90 & 32.82 & 32.77 & 32.57 \\
\hline & $\mathrm{Q}_{\mathrm{M} \& \mathrm{~V}}$ & 34.88 & 33.89 & 33.25 & 33.31 & 33.23 & 33.07 \\
\hline \multirow{2}{*}{ SP08 } & $\mathrm{QV}_{\mathrm{V}}$ & 30.87 & 30.45 & 30.29 & 30.20 & 30.16 & 30.57 \\
\hline & $\mathrm{Q}_{\mathrm{M} \& \mathrm{~V}}$ & 33.28 & 32.60 & 32.34 & 32.25 & 32.47 & 32.62 \\
\hline \multirow{2}{*}{ SP09 } & $\mathrm{Q}_{\mathrm{V}}$ & 32.35 & 32.16 & 32.33 & 32.23 & 32.24 & 32.25 \\
\hline & $\mathrm{Q}_{\mathrm{M} \& \mathrm{~V}}$ & 33.86 & 33.26 & 33.27 & 33.26 & 33.29 & 33.28 \\
\hline \multirow{2}{*}{ SP10 } & $\mathrm{Q}_{\mathrm{V}}$ & 29.30 & 28.99 & 28.93 & 29.00 & 29.05 & 29.22 \\
\hline & $\mathrm{Q}_{\mathrm{M} \& \mathrm{~V}}$ & 34.11 & 32.85 & 32.03 & 32.30 & 32.32 & 32.62 \\
\hline \multirow{2}{*}{ SP11 } & $\mathrm{Q}_{\mathrm{V}}$ & 32.87 & 32.76 & 32.64 & 32.60 & 32.79 & 32.31 \\
\hline & $\mathrm{Q}_{\mathrm{M} \& \mathrm{~V}}$ & 33.54 & 33.20 & 33.06 & 33.21 & 33.41 & 32.97 \\
\hline \multirow{2}{*}{ SP12 } & $\mathrm{Q}_{\mathrm{V}}$ & 32.22 & 32.26 & 33.04 & 32.94 & 33.11 & 32.86 \\
\hline & $\mathrm{Q}_{\mathrm{M} \& \mathrm{~V}}$ & 35.67 & 33.52 & 33.21 & 33.31 & 33.68 & 33.42 \\
\hline \multirow{2}{*}{ SP13 } & $\mathrm{Q}_{\mathrm{V}}$ & 32.34 & 32.34 & 32.51 & 32.41 & 32.38 & 32.47 \\
\hline & $\mathrm{Q}_{\mathrm{M} \& \mathrm{~V}}$ & 35.57 & 33.29 & 32.81 & 32.77 & 32.76 & 33.17 \\
\hline \multirow{2}{*}{ SP14 } & $\mathrm{Q}_{\mathrm{V}}$ & 32.39 & 32.68 & 32.40 & 32.42 & 32.38 & 32.65 \\
\hline & $\mathrm{Q}_{\mathrm{M} \& \mathrm{~V}}$ & 35.86 & 33.94 & 32.87 & 32.87 & 33.02 & 33.33 \\
\hline \multirow{2}{*}{ SP15 } & $\mathrm{Q}_{\mathrm{V}}$ & 32.44 & 32.45 & 32.65 & 32.64 & 32.77 & 32.47 \\
\hline & $\mathrm{Q}_{\mathrm{M} \& \mathrm{~V}}$ & 36.59 & 33.95 & 33.03 & 33.30 & 33.38 & 33.24 \\
\hline \multirow{2}{*}{ SP16 } & $\mathrm{Q}_{\mathrm{V}}$ & 32.10 & 32.01 & 32.04 & 32.27 & 32.00 & 31.94 \\
\hline & $\mathrm{Q}_{\mathrm{M} \& \mathrm{~V}}$ & 33.66 & 33.08 & 33.08 & 33.44 & 33.18 & 33.10 \\
\hline
\end{tabular}

\section{REFERENCES}

[1] A. Kondoz, Digital speech coding for low bit rate communication systems, New Jersey: JohnWiley \& Sons, 2004.

[2] Z. H. Perić, M. R. Dinčić, D. B. Denić, A. V. Jocic, "Forward adaptive logarithmic quantizer with new lossless coding method for Laplacian source", Wireless Personal Communications, vol. 59, no. 4, pp. 625-641, 2011.

[3] A.Spanias, T. Painter, V. Atti, Audio Signal Processing and Coding, New Jersey: JohnWiley \& Sons, 2007.

[4] M. Nakayama, N. Shigekawa, T. Yokouchi, S. Ishimitsu, "Frame-by-Frame Speech Signal Processing and Recognition for FPGA Devices", Sensors for Everyday Life, pp. 87-102, 2017. 
[5] N. S. Jayant, P. Noll, Digital Coding of Waveforms - principles and applications to speech and video, Englewood Cliffs, Prentice-Hall, 1984.

[6] W.C. Chu, Speech coding algorithms, foundation and evolution of standardized coders, New Jersey, John Wiley \& Sons, 2003.

[7] J. D. Markel, A. H. Gray Jr, "Linear Prediction of Speech", Series: Communication and Cybernetics, vol. 12, Springer-Verla, 1976.

[8] Jerry D. Gibson, "Speech Compression”, Information, ISSN 2078-2489, MDPI, vol. 7, no. 2, $32,2016$. doi:10.3390/info7020032.

[9] K. Sayood, Introduction to Data Compression, Fourth Ed., Elsevier, 2012.

[10] A. Gersho, R. M. Gray, Vector Quantization and Signal Compression, Kluwer Academic Publishers, 1992.

[11] ITU-T Test Signals for Telecommunication Systems, Test Vectors Associated to Rec. ITU-T P.50, 2015. [Online]. Available: http://www.itu.int/ITU-T/dbase.

[12] S. Na, On the support of fixed-rate minimum mean-squared error scalar quantizers for a Laplacian source, IEEE Transactions on Information Theory, vol. 50, no. 5, pp. 937-944, 2004.

[13] S. Na, D. Neuhoff, On the support of MSE-optimal fixed-rate scalar quantizers, IEEE Transactions on Information Theory, vol. 47, no. 7, 2972 - 2982, 2001.

[14] S. Gazor and W. Zhang, "Speech probability distribution," IEEE Signal Process. Lett., , vol. 10, no. 7 , pp. 204-207, Jul. 2003.

[15] Z. H. Perić, M. R. Dinčić, M. D. Petković, "Design of a hybrid quantizer with variable length code", Fundamenta informaticae, vol. 98, no. 2-3, pp. 233-256, 2010.

[16] M. Savić, Z. Perić, M. Dinčić, "Design of forward adaptive uniform quantizer for discrete input samples for Laplacian source", Elektronika ir Elektrotechnika, vol. 105, no. 9, pp. 73-76, 2010.

[17] J. C. Lagarias, J. A. Reeds, M. H. Wright, P. E. Wright, "Convergence Properties of the Nelder-Mead Algorithm in Low Dimensions", AT\&T Bell Laboratories Tech. Rep. Murray Hill, NJ, 1995.

[18] J. A. Nelder, R. Mead, "A Simplex Method for Function Minimization”, The Computer Journal, vol. 7 , no. 4, pp. 308-313, 1965.

[19] Z Peric, J Nikolic, "Asymptotic analysis of switched uniform polar quantization for memoryless Gaussian source”, IEEE Signal Processing Letters, Vol. 20, no. 1, pp. 75-78, 2013.

[20] Z Perić, J Nikolić, "An adaptive waveform coding algorithm and its application in speech coding", Digital Signal Processing, Vol.22, no.1, pp. 199-209, 2012.

[21] L Velimirović, Z Perić, J Nikolić, M. Stanković, "Design of compandor quantizer for Laplacian source for medium bit rate using spline approximations", Facta universitatis-series: Electronics and Energetics, Vol.2, no. 1, pp. 81-92, 2012.

[22] Z Peric, J Nikolic, A Mosic, S Panic, "A switched-adaptive quantization technique using $\mu$-law quantizers" Information Technologu and Control, vol. 39, no 4, pp 317-320, 2010. 